\title{
Usando heurísticas de Nielsen para avaliar objetos de aprendizagem e softwares educacionais: um estudo exploratório na área de Matemática para ensino superior
}

\author{
Karen Selbach Borges ${ }^{1}$, Clevi Elena Rapkiewicz ${ }^{2}$, Ana Carolina Feijó ${ }^{2}$ \\ ${ }^{1}$ Instituto Federal de Ciência e Tecnologia do Rio Grande do Sul - IFRS - Campus \\ Porto Alegre \\ ${ }^{2}$ Universidade Federal do Rio Grande do Sul \\ karen.borges@poa.ifrs.edu.br, clevirap@gmail.com, acfeijodinf.ufrgs.br
}

\begin{abstract}
Nowadays several repositories of learning objects (LOs) are available in Brazil. However, only the content of few of them has been examined, especially from the standpoint of usability. The goal of this paper is to verify the suitability of applying usability criteria in the evaluation of LOs. As a base exploratory study, learning objects in the area of Mathematics for higher education were adopted.
\end{abstract}

Resumo. Atualmente é possível encontrar diversos repositórios de objetos de aprendizagem (OAs) no Brasil. No entanto, poucos desses repositórios tiveram o seu conteúdo analisado, principalmente sob o foco da avaliação de usabilidade Nesse contexto o presente artigo verifica a possibilidade de aplicação de critérios de usabilidade na avaliação de OAs. Para tanto, adotou-se como base um estudo exploratório realizado com objetos de aprendizagem na área de Matemática para o ensino superior.

\section{Introdução}

Entre os recursos de Tecnologias de Informação e Comunicação (TIC) possíveis de serem utilizados no processo de ensino e aprendizagem encontram-se os objetos de aprendizagem (OAs). OAs podem ser definidos como componentes instrucionais que podem ser reutilizados em diferentes contextos (WILLEY, 2002). Outra definição para OA é "qualquer recurso, suplementar ao processo de aprendizagem, que pode ser reusado para apoiar a aprendizagem" (TAROUCO, FABRE e TAMUSIUNAS, 2003, p. 2). Espera-se que os OAs integrem diversas mídias (vídeo, som, texto, imagem) que, usadas com estratégias pedagógicas adequadas permitam resultados significativos na educação.

Uma das etapas necessárias para a incorporação de OAs no processo de ensino e aprendizagem, na visão dos autores desse artigo, é a escolha de quais objetos usar. Para que sejam selecionados objetos, é preciso primeiro localizá-los e, sem seguida, identificar quais possuem as características desejadas pelo usuário, seja professor ou aluno. A localização de objetos é feita, normalmente, através de repositórios, isto é, banco de dados que, além de armazenarem os objetos em si (NUNWA, 2004) provêem recursos de modo a facilitar a localização dos OAs. Tais recursos atuam como filtros e permitem localizar os OAs através de parâmetros. Normalmente as buscas são 
parametrizadas pelas palavras-chave (keywords) cadastradas, mas podem também ser refinadas para um determinado conteúdo, tecnologia, pedagogia, nível de interação, autoria, contribuições, entre outros. No entanto, consulta a partir da avaliação dos objetos contidos no repositório não tem sido comuns nos repositórios existentes no Brasil, conforme apontado na seção 2.

No intuito de colaborar com professores e alunos no processo de seleção de OAs como recursos educacionais, este artigo verifica a possibilidade de aplicação de critérios de usabilidade na avaliação de OAs. Segundo a ISO9241 usabilidade é a "capacidade que um sistema interativo oferece a seu usuário, em determinado contexto de operação, para a realização de tarefas de maneira eficaz, eficiente e agradável". Ou seja, em princípio a usabilidade forneceria critérios para seleção de objetos. Essa avaliação está inserida no contexto de uma metodologia de avaliação de objetos descrita na seção 3.

Na seção 4 é apresentado um estudo exploratório para análise da viabilidade de aplicação do arcabouço teórico definido para avaliação de objetos e na seção 5 são apresentadas algumas considerações finais.

\section{Repositórios Nacionais de OAs}

Diversos repositórios de OAs encontram-se disponíveis no Brasil. Alguns estão destacados na Tabela 1.

Tabela 1. Repositórios de OAS

\begin{tabular}{|l|l|l|}
\hline \multicolumn{1}{|c|}{ Nome } & \multicolumn{1}{|c|}{$\begin{array}{c}\text { Instituição } \\
\text { Mantenedora }\end{array}$} & \multicolumn{1}{c|}{ Descrição } \\
\hline $\begin{array}{l}\text { RIVED - Rede } \\
\text { Int.Virtual de Educação }\end{array}$ & $\begin{array}{l}\text { MEC - Ministério da } \\
\text { Educação }\end{array}$ & $\begin{array}{l}\text { Repositório de OA do projeto Fábrica } \\
\text { Virtual. }\end{array}$ \\
\hline $\begin{array}{l}\text { BIOE - Banco } \\
\text { Internacional de Objetos } \\
\text { Educacionais }^{2}\end{array}$ & $\begin{array}{l}\text { MEC - Ministério da } \\
\text { Educação }\end{array}$ & $\begin{array}{l}\text { Repositório com diversos tipos de recursos } \\
\text { educacionais, tai como vídeo, som, } \\
\text { animação, simulação, etc. }\end{array}$ \\
\hline $\begin{array}{l}\text { LabVirt - Laboratório } \\
\text { Didático Virtual }\end{array}$ & $\begin{array}{l}\text { USP - Universidade de } \\
\text { São Paulo }\end{array}$ & $\begin{array}{l}\text { Repositório com diversos tipos de recursos } \\
\text { educacionais incluindo artigos, discussões, } \\
\text { fórum, etc. }\end{array}$ \\
\hline $\begin{array}{l}\text { CESTA - Coletânea de } \\
\text { Entidades de Suporte ao } \\
\text { uso de Tecnologia na } \\
\text { Aprendizagem }\end{array}$ & $\begin{array}{l}\text { UFRGS - Universidade } \\
\text { Federal do Rio Grande do } \\
\text { Sul }\end{array}$ & $\begin{array}{l}\text { Repositório com diversos recursos } \\
\text { multimídia, mas necessita de autenticação } \\
\text { (login e senha) para a navegação. }\end{array}$ \\
\hline Portal do Professor & $\begin{array}{l}\text { MEC - Ministério da } \\
\text { Educação }\end{array}$ & $\begin{array}{l}\text { Repositório com recursos educacionais } \\
\text { diversos: áudio, vídeo, experimentos, } \\
\text { mapas, animação e simulação. }\end{array}$ \\
\hline Proativa ${ }^{6}$ & $\begin{array}{l}\text { UFC - Universidade } \\
\text { Federal do Ceará }\end{array}$ & $\begin{array}{l}\text { Repositório com objetos na área de } \\
\text { Biologia, Física, Ciências, Química, } \\
\text { Matemática Linguagem. }\end{array}$ \\
\hline
\end{tabular}

\footnotetext{
${ }^{1}$ http://www.rived.mec.gov.br

${ }^{2}$ http://objetoseducacionais2.mec.gov.br/

${ }^{3}$ http://www.labvirtq.fe.usp.br/indice.asp

${ }^{4}$ http://cesta.cinted.ufrgs.br/

${ }^{5} \mathrm{http} / / /$ portaldoprofessor.mec.gov.br/index.html

${ }^{6} \mathrm{http} / / / \mathrm{www}$. proativa.vdl.ufc.br/oa.php
} 
O portal do RIVED é um projeto sem continuidade, mas que ainda oferece consulta aos objetos produzidos com financiamento daquele projeto. Não há, no entanto, avaliação dos objetos em si, o que é possível fazer em alguns casos é estabelecer contato com a equipe de criação dando algum tipo de feedback sobre o objeto. Entretanto, o usuário não tem acesso a comentários já feitos por outros usuários, uma vez que a forma de contato é unidirecional, do usuário para a equipe autora.

No BIOE também não há consulta por avaliação, pelo menos não no nível de usuário comum, isto é, sem login e senha de acesso. Além da consulta por filtros para localizar OAs, o que se encontra disponível é consulta quantitativa sobre o tipo de mídia usada (vídeo, som, imagem), sobre o tipo de recurso usado (simulação, experimento prático, software educacional, hipertexto). Essas consultas quantitativas também podem ser feitas por nível de ensino e por área.

No LabVirt é possível adicionar comentários especificando se é técnico ou pedagógico. Também é possível apresentar sugestões como um todo para o repositório. Outra opção é participar de um fórum. No entanto, como no caso do RIVED, não há disponibilização dos comentários feitos por outros usuários a respeito dos recursos presentes no repositório.

No CESTA é possível fazer consulta básica por palavras-chave e uma pesquisa avançada com várias opções, incluindo nível de interatividade, tipo de recurso, entre outros. Não há no entanto qualquer consulta baseada na visão que usuários tenham dos objetos existentes no repositório.

No Portal do Professor, na pesquisa avançada ao final dos vários filtros possíveis para localização de recursos, encontra-se a possibilidade de classificação por quantidade de comentários feitos sobre o recurso. São comentários textuais que não seguem critérios previamente definidos, mas são importantes por serem feitos por pares, isto é, por outros professores que já usaram o recurso. Também é apresentada uma graduação do recurso em quantidade de estrelas, feita pelos usuários.

Finalmente, o PROATIVA além de não apresentar consulta através de filtros também não provê a possibilidade de avaliar o objeto ou de consultar avaliações feitas por pares.

Em resumo, dos cinco repositórios analisados o único que apresenta avaliação pelos pares, disponível para consulta, é o Portal do Professor. No entanto, são comentários livres que não necessariamente apontam critérios específicos, técnicos ou pedagógicos, que os objetos atendem ou não, ou atendem parcialmente. Os comentários possíveis de serem feitos no LabVirt estão categorizados em dimensão técnica e pedagógica, mas não são refinados e não estão disponibilizados para os usuários.

\section{Critérios e Metodologias para Avaliar OAs}

Daniel e Mohan (2004) apresentam uma metodologia de avaliação de objetos de aprendizagem (OA) baseada em duas dimensões, conforme a figura 1. 


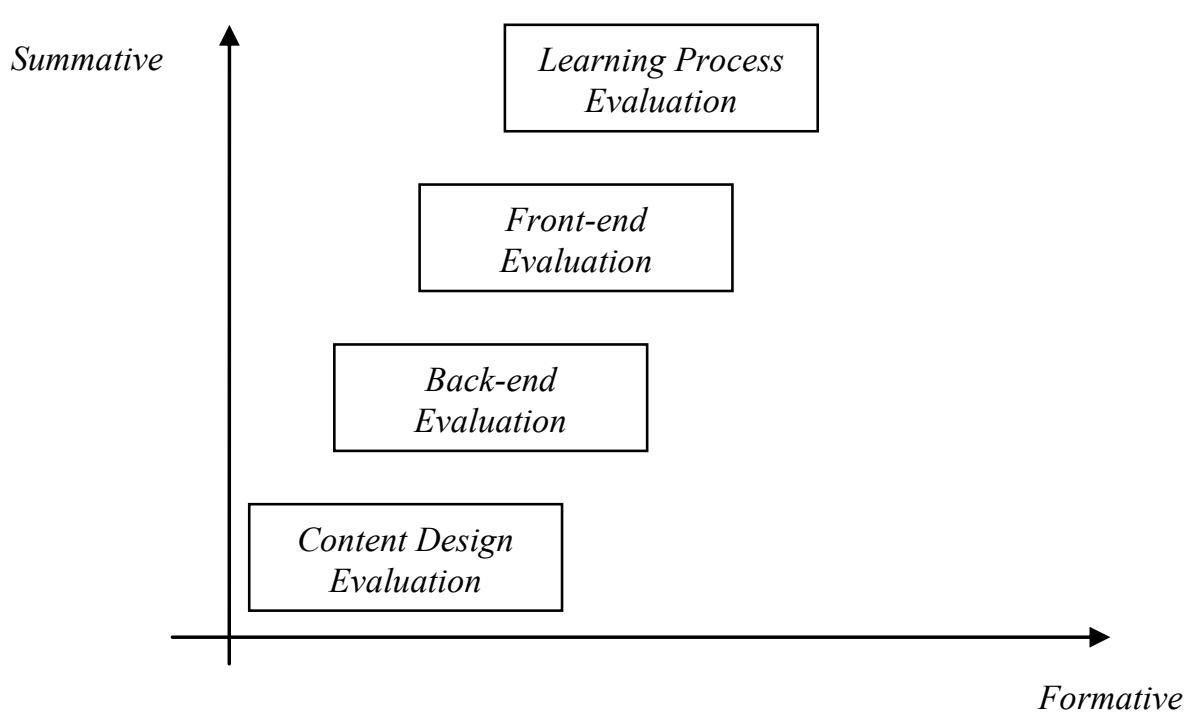

Figura 1. Processo Avaliativo de OA (adaptado de Daniel e Mohan (2004))

A dimensão somativa propõe uma avaliação incremental. Já a dimensão formativa indica que os resultados individuais das avaliações serão determinantes para a elaboração do conceito final da avaliação. Dentro destas dimensões, os autores propõem que a avaliação dos OAs leve em consideração aspectos relacionados às etapas de projeto, desenvolvimento e distribuição dos objetos. Assim, o processo de avaliação foca aspectos de (i) design de conteúdo; (ii) gerenciamento de repositórios e distribuição (back-end); (iii) apresentação e usabilidade (front-end) e (iv) processo de aprendizagem.

Considerando-se as limitações próprias de um estudo exploratório, optou-se por aplicar uma das fases do modelo, voltada para a interface. Assim, o presente trabalho aborda às questões relativas à avaliação do front-end dos OAs. Nesta etapa de avaliação, deve-se levar em consideração que, em situações onde o estudante possui acesso mínimo ao tutor, é necessário garantir que o OA seja intuitivo e seguro (no seu uso), forneça feedback constante, possua recursos de ajuda, além de uma interface agradável, contextualizada e estimulante. No mesmo sentido para o professor que, em muitas escolas, não conta com suporte específico de informática.

Estes aspectos remetem diretamente à avaliação de usabilidade. Uma maneira de avaliar a usabilidade de um sistema é através de heurísticas, das quais destacam-se as chamadas "Heurísticas de Nielsen" (1993), as quais são:

- Feedback: Informar continuamente o usuário sobre o que ele está fazendo.

- Falar a linguagem do usuário: Terminologia adequada ao usuário e não ao sistema. Buscar organizar as informações de acordo com o modelo mental do usuário.

- Saídas demarcadas: Possibilidade de uma tarefa ser cancelada ou uma operação ser desfeita voltando ao estado anterior. Ou seja, o usuário controla o sistema.

- Consistência: Fazer a mesma coisa sempre do mesmo jeito, isto é, a mesma operação deve ser apresentada sempre da mesma maneira para facilitar o reconhecimento. $\mathrm{O}$ efeito de uma ação deve ser sempre o mesmo. 
- Prevenir erros: Ter clara as situações que mais provocam erros e modificar a interface para que tais erros não ocorram.

- Independência da memória do usuário: A interface deve propiciar que o usuário faça suas escolhas sem necessidade de lembrar comandos específicos.

- Atalhos: Fornecer atalhos para acesso a informações com muita profundidade na árvore de navegação. Fornecer abreviações, teclas de função, etc, para usuários experientes poderem executar as funções de modo mais rápido.

- Diálogos simples e naturais: A informação apresentada ao usuário deve ser adequada ao momento e contexto, sem excesso de informação nem falta. $\mathrm{O}$ acesso às operações deve ser compatível com o modo como o usuário interage.

- Boas mensagens de erros: Mensagens de erro que ajudem a resolver problemas, não punindo ou intimidando o usuário, usando linguagem clara e sem códigos.

- Ajuda e documentação: O sistema deveria ser de tal forma intuitivo que a necessidade de ajuda ou documentação seria dispensável. Mas sendo necessária deve estar facilmente acessível.

Estes critérios foram utilizados durante o processo exploratório a seguir descrito.

\section{Estudo Exploratório: analisando OAs e softwares educacionais de Matemática para o Ensino Superior}

A atividade de investigação iniciou com a escolha do repositório de objetos. O Banco Internacional de Objetos Educacionais (BIOE) foi escolhido por constituir o mais amplo e completo repositório nacional de objetos educacionais. Mantido pelo Ministério da Educação, oferece acesso a objetos educacionais voltados, desde a educação infantil, até o ensino superior (figura 2), abrangendo as mais diversas áreas, das artes visuais à matemática.

A área escolhida para serem feitas as análises foi a matemática voltada para $\mathrm{o}$ ensino superior. Essa escolha foi feita considerando as dificuldades que alunos de ensino superior tem com essa área do conhecimento (PALIS, 2009) e por ser um conteúdo que encontra-se presente em vários cursos de graduação. Também colaborou para a definição da área de análise a formação dos pesquisadores envolvidos nesse projeto (Computação).

Finalmente, um terceiro critério de seleção foi a quantidade de objetos existentes: o BIOE conta com mais de 800 objetos educacionais de matemática voltados para o ensino superior. Ao analisar os recursos presentes no BIOE, verificou-se que eventualmente alguns poderiam ser entendidos mais como softwares educacionais do que propriamente objetos como no caso do Poly. Optamos por incluir na análise também esses casos, por pelo menos duas razões:

i) são recursos disponíveis num repositório destinado a objetos;

ii) concordância com Santanché et al (2008) que, ao discutir características e conceitos de Oas, apontam o caráter vago da definição que permite considerar muitas coisas como objetos e ao mesmo tempo criticam definições muito restritivas que poderiam excluir bons objetos. Os autores tem uma visão crítica 
até mesmo do que é software em si, enfatizando que atualment há deslocamento da noção antiga de software como código executável para uma amplitude de possibilidades que inclui páginas Web, animações, entre outras.

Optou-se, pois, por não separar as duas categorias, selecionando vinte objetos ou softwares educacionais, priorizando aqueles que apresentavam maior nível de interatividade.

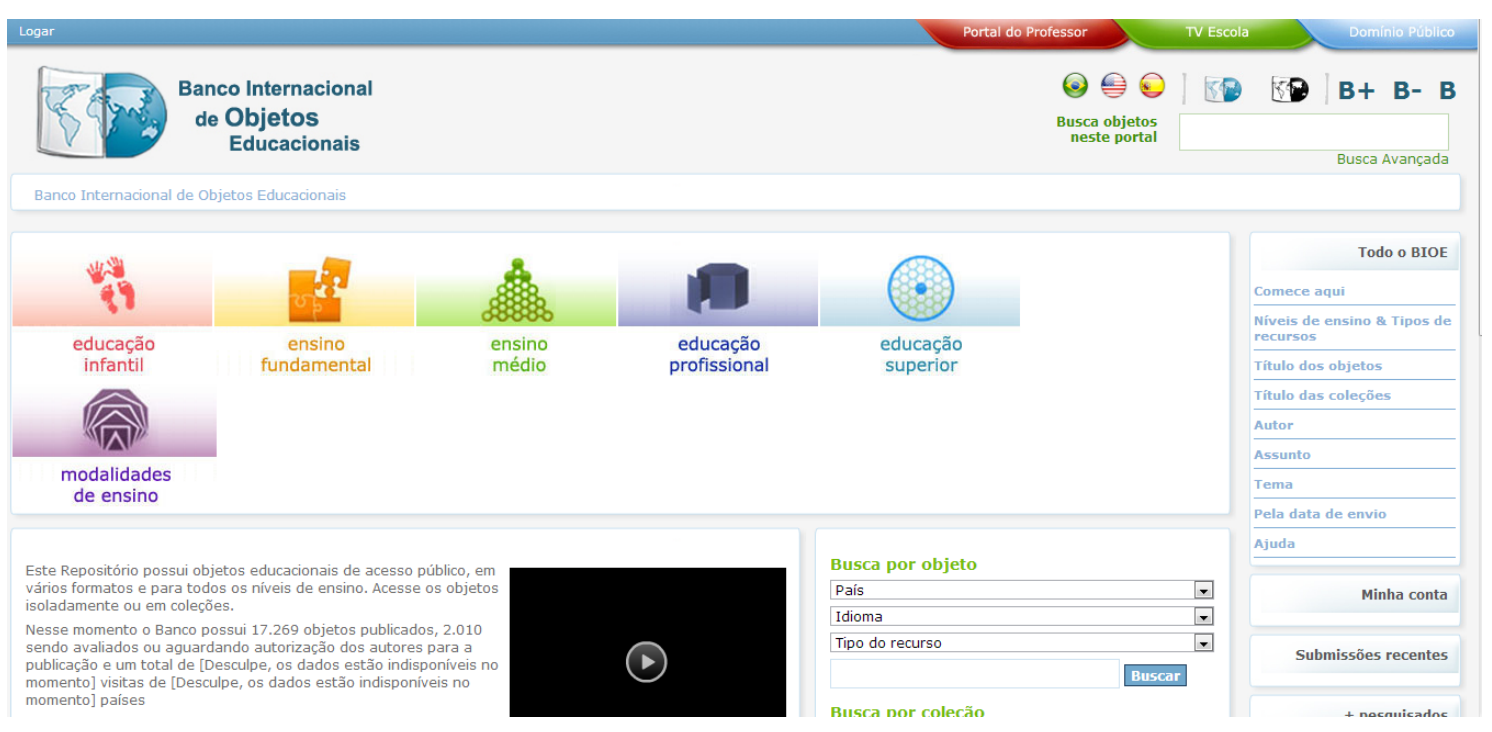

Figura 2. Tela inicial do BIOE.

Após o estudo exploratório sobre os vinte recursos selecionados, constatou-se que doze não possuíam condições de serem avaliados em função das heurísticas de Nielsen, pois não apresentavam recursos interativos suficientes. Os oito objetos restantes são apresentados, de forma sintetizada, através da Tabela 2:

Tabela 2. Comparativo entre OAs

\begin{tabular}{|c|c|c|c|c|}
\hline & Título & Objetivos & Prós & Contras \\
\hline 1 & Acogeo & $\begin{array}{l}\text { Apresenta um conjunto de } \\
\text { programas que permitem } \\
\text { visualizar os conceitos de } \\
\text { geometria diferencial no } \\
\text { estudo local das curvas e } \\
\text { superfícies regulares }\end{array}$ & $\begin{array}{lr}\text { Organizado } & \text { de } \\
\text { maneira } & \text { que } \\
\text { qualquer } & \\
\text { usuário, } \\
\text { experiente } \\
\text { não, ou } \\
\text { mexer ronsiga } \\
\text { causar } \\
\text { erros }\end{array}$ & $\begin{array}{l}\text { Ações } \\
\text { destrutivas } \\
\text { perante erros } \\
\text { cometidos pelo } \\
\text { usuário, não } \\
\text { apresenta boas } \\
\text { mensagens de } \\
\text { erro, dificuldade } \\
\text { na entrada de } \\
\text { dados }\end{array}$ \\
\hline 2 & Cálculo Numérico & $\begin{array}{l}\text { Objeto voltado a } \\
\text { implementação de funções } \\
\text { e métodos numéricos } \\
\text { vistos nas disciplinas de } \\
\text { cálculo numérico }\end{array}$ & $\begin{array}{l}\text { Presença de } \\
\text { feedbacks e } \\
\text { inúmeros atalhos } \\
\text { e independe da } \\
\text { memória do } \\
\text { usuário }\end{array}$ & $\begin{array}{l}\text { Menu com } \\
\text { informações } \\
\text { demais, } \\
\text { possivelmente } \\
\text { causando o } \\
\text { desnorteamento } \\
\text { do usuário }\end{array}$ \\
\hline
\end{tabular}




\begin{tabular}{|c|c|c|c|c|}
\hline 3 & Complex Numbers & $\begin{array}{l}\text { O objetivo desse objeto é } \\
\text { avaliar equação } \\
\text { complexas, podendo achar } \\
\text { a n-ésima raíz e mostrar } \\
\text { gráficos dessas equações }\end{array}$ & $\begin{array}{l}\text { Bom sistema de } \\
\text { ajuda }\end{array}$ & $\begin{array}{l}\text { Design não } \\
\text { atraente, não } \\
\text { trata de erros } \\
\text { previamente, } \\
\text { péssimas } \\
\text { mensagens de } \\
\text { erro, usuário não } \\
\text { está sempre no } \\
\text { controle }\end{array}$ \\
\hline 4 & Poly & $\begin{array}{l}\text { Permite construir e } \\
\text { analisar poliedros e sólidos } \\
\text { tridimensionais, } \\
\text { possibilitando } \\
\text { planarização dos mesmos. }\end{array}$ & $\begin{array}{l}\text { Bom sistema de } \\
\text { ajuda e trata } \\
\text { previamente } \\
\text { casos de erro }\end{array}$ & $\begin{array}{lr}\text { Usuário não } & \text { está } \\
\text { sempre } & \text { no } \\
\text { controle } & \text { do } \\
\text { programa } & \end{array}$ \\
\hline 5 & Taylor Series & $\begin{array}{l}\text { Apresenta as primeiras } 20 \\
\text { (vinte) parcelas da série de } \\
\text { Maclaurin. }\end{array}$ & 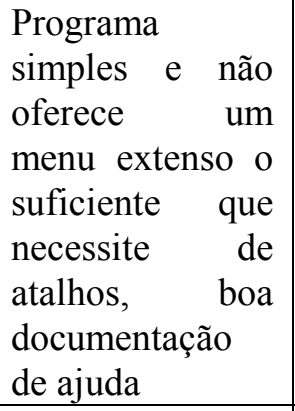 & $\begin{array}{l}\text { Requer um } \\
\text { pouco de } \\
\text { conhecimento } \\
\text { sobre o assunto } \\
\text { tratado para } \\
\text { melhor manuseio } \\
\text { do programa }\end{array}$ \\
\hline 6 & Wingeon & $\begin{array}{l}\text { Permite } \\
\text { geométricas no plano e } \\
\text { espaço para o ensino de } \\
\text { geometria. }\end{array}$ & $\begin{array}{l}\text { Interface de fácil } \\
\text { acesso } \\
\text { concordando } \\
\text { com o quesito de } \\
\text { flexibilidade do } \\
\text { sistema }\end{array}$ & $\begin{array}{l}\text { Mau tratamento } \\
\text { de situações de } \\
\text { erros }\end{array}$ \\
\hline 7 & $\begin{array}{l}\text { Are You Ready for } \\
\text { Calculus I? }\end{array}$ & $\begin{array}{l}\text { A partir de pequenos testes } \\
\text { de perguntas e respostas, o } \\
\text { objeto analisa se o usuário } \\
\text { estaria pronto ou não para } \\
\text { cursar a disciplina de } \\
\text { Cálculo I, fornecendo no } \\
\text { final bibliografia de estudo } \\
\text { de acordo com os possíveis } \\
\text { erros do usuário. }\end{array}$ & $\begin{array}{l}\text { Concorda com } \\
\text { todos critérios } \\
\text { de usabilidade }\end{array}$ & Nenhum \\
\hline 8 & Francisvest & $\begin{array}{l}\text { Programa calcula } \\
\text { financiamentos a partir das } \\
\text { prestações, taxas de juros e } \\
\text { número de meses. }\end{array}$ & $\begin{array}{l}\text { Entradas claras, } \\
\text { boa } \\
\text { documentação } \\
\text { de ajuda }\end{array}$ & $\begin{array}{l}\text { Não apresenta } \\
\text { introdução } \\
\text { explicando o que } \\
\text { o objeto faz, } \\
\text { arredonda } \\
\text { valores sem } \\
\text { pedir } \\
\text { confirmação } \\
\text { para o usuário }\end{array}$ \\
\hline
\end{tabular}


A tabela 3, apresenta a análise destes mesmos oito recursos, sob a perspectiva das heurísticas. Leia-se:

- $\mathrm{N}$ : não atende a heurística;

- S: atende a heurística;

- NA: não se aplica. Caso em que não foi possível identificar recurso do software que pudesse ser avaliado conforme a heurística em questão.

Tabela 3. Resultado da Análise Exploratória

\begin{tabular}{|l|l|l|l|l|l|l|l|l|}
\hline \multicolumn{1}{|c|}{ Número do Objeto } & $\mathbf{1}$ & $\mathbf{2}$ & $\mathbf{2}$ & $\mathbf{4}$ & $\mathbf{5}$ & $\mathbf{6}$ & $\mathbf{7}$ & $\mathbf{8}$ \\
\hline Feedback & $\mathrm{N}$ & $\mathrm{S}$ & $\mathrm{N}$ & $\mathrm{N}$ & $\mathrm{N}$ & $\mathrm{N}$ & $\mathrm{S}$ & $\mathrm{NA}$ \\
\hline Falar a linguagem do usuário & $\mathrm{S}$ & $\mathrm{S}$ & $\mathrm{S}$ & $\mathrm{S}$ & $\mathrm{S}$ & $\mathrm{S}$ & $\mathrm{S}$ & $\mathrm{S}$ \\
\hline Saídas demarcadas & $\mathrm{S}$ & $\mathrm{S}$ & $\mathrm{N}$ & $\mathrm{N}$ & $\mathrm{S}$ & $\mathrm{S}$ & $\mathrm{S}$ & $\mathrm{N}$ \\
\hline Consistência & $\mathrm{S}$ & $\mathrm{S}$ & $\mathrm{S}$ & $\mathrm{S}$ & $\mathrm{S}$ & $\mathrm{S}$ & $\mathrm{S}$ & $\mathrm{S}$ \\
\hline Prevenir erros & $\mathrm{N}$ & $\mathrm{N}$ & $\mathrm{N}$ & $\mathrm{S}$ & $\mathrm{N}$ & $\mathrm{N}$ & $\mathrm{S}$ & $\mathrm{S}$ \\
\hline Independência da memória do usuário & $\mathrm{S}$ & $\mathrm{S}$ & $\mathrm{N}$ & $\mathrm{S}$ & $\mathrm{S}$ & $\mathrm{N}$ & $\mathrm{S}$ & $\mathrm{S}$ \\
\hline Atalhos & $\mathrm{S}$ & $\mathrm{S}$ & $\mathrm{N}$ & $\mathrm{N}$ & $\mathrm{N}$ & $\mathrm{S}$ & $\mathrm{S}$ & $\mathrm{NA}$ \\
\hline Diálogos simples e naturais & $\mathrm{S}$ & $\mathrm{S}$ & $\mathrm{S}$ & $\mathrm{S}$ & $\mathrm{S}$ & $\mathrm{S}$ & $\mathrm{S}$ & $\mathrm{N}$ \\
\hline Boas mensagens de erro & $\mathrm{N}$ & $\mathrm{N}$ & $\mathrm{N}$ & $\mathrm{N}$ & $\mathrm{N}$ & $\mathrm{N}$ & $\mathrm{S}$ & $\mathrm{NA}$ \\
\hline Ajuda e documentação & $\mathrm{N}$ & $\mathrm{S}$ & $\mathrm{S}$ & $\mathrm{S}$ & $\mathrm{S}$ & $\mathrm{S}$ & $\mathrm{S}$ & $\mathrm{S}$ \\
\hline
\end{tabular}

Esta tabela mostra a utilidade de aplicar as heurísticas de Nielsen para avaliação da interface na medida em que permite apontar pontos fortes e fracos que permitem orientar tanto os usuários na seleção de objetos quanto desenvolvedores para que atentem mais para determinadas características. Por exemplo, permite detacar, pela amostra analisada, como ponto forte os diálogos simples e naturias, mas como pontos fracos ausência ou dificuldades no feedback e dificuldades na prevenção de erros.

\section{Considerações Finais}

Conforme já apontado, os cinco repositórios de objetos de aprendizagem analisados não contemplam a disponibilização de avaliação dos recursos armazenados feito por pares de forma sistemática e baseada em critérios. Há alguma aproximação no Portal do Professor, aproximação esta, no entanto, que pode ser estruturada para atender critérios que, por exemplo, justifiquem as estrelas atribuídas.

O estudo exploratório apresentado nesse artigo utilizou heurísticas de Nielsen para avaliar a usabilidade de uma amostra de OAs de Matemática para o Ensino Superior disponíveis no BIOE. O estudo feito permite afirmar que é possível utilizar as heurísticas de Nielsen no processo avaliativo. Essas heurísticas poderiam ser associadas aos repositórios, permitindo aos usuários avaliarem o recurso, atribuindo algum tipo de classificação (como as estrelas do Portal do Professor) de acordo com as respostas dadas. Outras dimensões, além da usabilidade, poderiam ser acrescentadas, como a funcionalidade e a acessibilidade.

Um fator limitante para o uso das heurísticas é o grau de interatividade tanto dos objetos quanto dos softwares educacionais. Resta saber se a limitação, no caso, que deve ser apontada é do uso das heurísticas para análise ou se a limitação em si está no objeto ou software educacional, pois, dadas as múltiplas possibilidades de interação que a 
tecnologia permite, é discutível o uso da mesma para produção de materiais não interativos, ainda que estes tenham seu espaço no processo de ensino e aprendizagem.

A análise da amostra de recursos do BIOE permite também apontar o uso das heurísticas de Nielsen como apoio a um bom projeto de interface. Afirmamos isso com base no conjunto de fatores cruciais que o estudo exploratório permitiu identificar para serem levados em conta enquanto denvolve-se um objeto educacional, os quais são:

- Atenção para deixar o programa o mais simples e conciso possível, tornando-o acessível tanto a usuários novatos quanto aos mais experientes;

- Tomar cuidado com o custo computacional que o programa necessita;

- Otimizar a organização espacial das informações de entrada e saída;

- Interfaces compostas por entradas simples, já que o contrário pode fazer com que o usuário fique perdido devido ao turbilhão de dados;

- Disposição inteligente de dados, sempre visando a facilidade de compreensão do usuário;

- Não realizar nenhuma ação destrutiva sem a consulta do usuário.

- Não depender de atributos do usuário, construir um objeto o mais amplo o possível, para que tanto o entendimento da sua interface quanto o de seu conteúdo seja compreendido por todos.

Considerando-se as limitações do estudo apresentado neste artigo, apresentamos como continuidade do mesmo executar um processo de avaliação heurística, com a participação de avaliadores técnicos e de domínio (pessoas que dominam o assunto), uma vez que, segundo Cybis (2007) "as avaliações heurísticas possuem a possibilidade de produzirem diagnósticos equivocados e sugestões de revisão superficiais. Tais fatos decorrem da falta de conhecimento mais aprofundado tanto sobre o contexto de uso dos sistemas como sobre o contexto do projeto da interface".

\section{Referências}

CYBIS, Walter; BETIOL, Adriana H; FAUST, Richard. Ergonomia e Usabilidade: Conhecimentos, Métodos e Aplicações. Editora Novatec, São Paulo, 2007.

DANIEL, B. ; MOHAN, P. A Model for Evaluating Learning Objects. In: Proceedings of the IEE International Conference on Advanced Learning Technologies (ICALI'04).

ISO9241-11. Ergonomic requirements for office work with visual display terminals (VDTs) - Part 11: Guidance on usability. Disponível em http://www.idemployee.id.tue.nl/g.w.m.rauterberg/lecturenotes/ISO9241part11.pdf. Acesso em abril de 2009.

NIELSEN, J. Usability Engineering. Academic Press, Cambridge, MA. 1993.

NUNES, C., Objetos de Aprendizagem em Ação. Cadernos de Pesquisa Reflexões. NEA/FEA/USP, Vol 1, nº $1,2004$.

PALIS, G. I. R. Pesquisa sobre a própria prática no ensino superior de Matemática. In.: FROTA, M.C.R.; NASSER, L. (org.) Educação Matemática no Ensino Superior: pesquisas e debates. Recife, PE:SBEM, 2009, p.203-221 
TAROUCO, L. M. R., FABRE, M. J. M. e TAMUSIUNAS, F. R. (2003), Reusabilidade de objetos educacionais. RENOTE v.1 n.1.

SANTANCHÉ, A.; LAGO, A.; DOURADO, P.; FERREIRA Filho, P. Ferramentas e ambientes para objetos de aprendizagem. XXVIII Simpósio Brasileiro de Informática na Educação. 2008. Minicurso.

WILLEY, D., Connecting learning objects to instructional design theory: A definition, a metaphor, and a taxionomy, 2002. Disponível em:<http://reusability.org/read/chpters/wiley.doc>. Acesso em: 10 maio. 2012. 